\title{
Symmetry breaking of quantum droplets in a dual-core trap
}

\author{
Bin Liu ${ }^{1}$, Hua-Feng Zhang ${ }^{2}$, Rong-Xuan Zhong ${ }^{1}$, Xi-Liang Zhang ${ }^{1}$, Xi-Zhou \\ Qin $^{1}$, Chunqing Huang ${ }^{1}$, and Yong-Yao $\mathrm{Li}^{1,3 *}$ and Boris A. Malomed ${ }^{3}$ \\ ${ }^{1}$ School of Physics and Optoelectronic Engineering, Foshan University, Foshan 528000, China \\ ${ }^{2}$ School of Physics and Optoelectronic Engineering, \\ Yangtze University, Jingzhou 434023, China and \\ ${ }^{3}$ Department of Physical Electronics, School of Electrical Engineering, Faculty of Engineering, \\ and Center for Light-Matter Interaction, Tel Aviv University, Tel Aviv 69978, Israel
}

\begin{abstract}
We consider the dynamical model of a binary bosonic gas trapped in a symmetric dual-core cigarshaped potential. The setting is modeled by a system of linearly-coupled one-dimensional GrossPitaevskii equations with cubic self-repulsive terms and quadratic attractive ones, which represent the Lee-Huang-Yang corrections to the mean-field theory in this geometry. The main subject is spontaneous symmetry breaking (SSB) of quantum droplets (QDs), followed by restoration of the symmetry, with respect to the identical parallel-coupled trapping cores, following the increase of the QD's total norm. The SSB transition and inverse symmetry-restoring one form a bifurcation loop, whose shape in concave at small values of the inter-core coupling constant, $\kappa$, and convex at larger $\kappa$. The loop does not exist above a critical value of $\kappa$. At very large values of the norm, QDs do not break their symmetry, featuring a flat-top shape. Some results are obtained in an analytical form, including an exact front solution connecting asymptotically constant zero and finite values of the wave function. Collisions between moving QDs are considered too, demonstrating a trend to merger into breathers.
\end{abstract}

PACS numbers: 03.75.Lm, 05.45.Yv

\section{INTRODUCTION}

Recently, a new type of self-bound quantum liquid states, in the form of three-dimensional (3D) droplets, was created experimentally in dipolar bosonic gases of dysprosium [1] and erbium 2], as well as in mixtures of two atomic states of ${ }^{39} \mathrm{~K}$ with contact interactions [3], following the theoretical proposal elaborated in Refs. 44] and [5]. These quantum droplets (QDs) are formed by the balance of attractive forces, which drive the collapse of the quantum gases in the mean-field approximation, and the repulsive force induced by quantum fluctuations around the mean-field states, which is represented by the quartic Lee-Huang-Yang (LHY) corrections [6] to the respective Gross-Pitaevskii equations (GPEs) with the usual cubic terms. In the dysprosium and erbium gases, the attractive force is generated by the dipole-dipole interactions, as it was analyzed in detail 7 15], while in the binary mixture it is provided by the inter-component attraction, which can be made slightly stronger than the intra-component repulsion by means of the Feshbach resonance, see further details in Refs. [16 23. Recently, the formation of QDs in Bose-Fermi mixture under the action of the spin-orbit coupling (SOC) [24], and a possibility to create similarly built photonic droplets [25] have also been predicted.

QDs are made of extremely dilute quantum fluids [26]. The droplets may be considered as soliton-like objects, with the unique property of stability in $2 \mathrm{D}$ and $3 \mathrm{D}$

*Electronic address: yongyaoli@gmail.com geometries, where usual nonlinear models give rise to solitons that are subject to strong instabilities [27, 28] [an exception is provided by pairs of GPEs with SOC terms, which predict absolutely stable 2D solitons, i.e., the system's ground states 29], and metastable 3D ones [30]]. Accordingly, stable QDs offer potential use in various applications, such as matter-wave interferometry [31-33] and manipulations of quantum information 34]. Furthermore, it was recently predicted that 2D QDs (whose effective nonlinearity is different from the abovementioned quartic form, amounting to cubic terms multiplied by a logarithmic factor [5]) with embedded vorticity $S=1,2,3, \ldots$ may be stable too, up to $S=5$ [35]. A related result is the stability of $2 \mathrm{D}$ QDs of the mixed-mode type (mixing vortical and zero-vorticity constituents), formed by the SOC effect 36. Full 3D QDs with embedded vorticity $S=1$ and 2 have also been predicted to have stability domains in the respective parameter space [37].

One of fundamental aspects of the soliton phenomenology is spontaneous symmetry breaking (SSB) of selftrapped modes in symmetric two-component systems. In particular, the SSB of optical solitons was considered in various settings 38 45], including coupled lasers [46, 47] and metamaterials [48] (see also a collection of articles on this topic [49], and a review in Ref. [50]). Applications of this effect, such as design of power-switch devices based on soliton light propagation in fibers, were proposed [41, 50]. In Bose-Einstein condensates (BECs), $\mathrm{SSB}$ of matter-wave solitons has also been considered in many configurations 50 57], but not, as yet, for QDs. In this work, we address effectively one-dimensional QDs in the binary bosonic gas loaded in a symmetric double-core 
cigar-shaped potential. Unlike the usual SSB mechanism for matter-wave solitons, which is induced by mean-field interactions, the SSB of QDs in this system is driven by the interplay of the mean-field and LHY terms.

The rest of the paper is structured as follows. The model is introduced in Sec. II, where some analytical results are presented too, such as an exact solution for a front interpolating between zero and an asymptotically constant wave function. Basic numerical results for the $\mathrm{SSB}$ of QDs are reported in Sec. III, which, in addition, includes some approximate analytical results related to the numerical ones. Collisions of two-component QDs are addressed in Sec. IV. The paper is concluded by Sec. $\mathrm{V}$.

\section{THE MODEL}

The system under the consideration is sketched in Fig. 11. QDs, which are formed in the binary bosonic gas, are trapped in the nearly-1D symmetric double-core potential, assuming, as usual, that the wave-function components of the two species of the binary condensate are equal in each core. Then, the system of linearly-coupled GPEs, including the LHY terms, are written in the scaled form as [5, 18]:

$$
\begin{aligned}
& i \partial_{t} \Psi_{1}=-\frac{1}{2} \partial_{x x} \Psi_{1}+g\left|\Psi_{1}\right|^{2} \Psi_{1}-\left|\Psi_{1}\right| \Psi_{1}-\kappa \Psi_{2}, \\
& i \partial_{t} \Psi_{2}=-\frac{1}{2} \partial_{x x} \Psi_{2}+g\left|\Psi_{2}\right|^{2} \Psi_{2}-\left|\Psi_{2}\right| \Psi_{2}-\kappa \Psi_{1},
\end{aligned}
$$

where $g \sim\left(g_{+-}+\sqrt{g_{++} g_{--}}\right) / \sqrt{g_{++g_{--}}}>0$ is the effective coefficient of the cubic repulsion [18] $\left(g_{++,--}\right.$and $g_{+,-}$are, respectively, strengths of the self- and crossinteraction of the two components), and $\kappa>0$ is the hopping rate which couples the parallel cores. By means of additional rescaling, we fix $g \equiv 1$ in Eq. (11), making $\kappa$ the single control parameter. The competition of the self-repulsive cubic and attractive quadratic terms in Eq. (1) determines the formation of QDs in this setting [18]. Previously, a dual-core model with the competition of cubic self-attraction and quintic repulsion in each core was introduced in optics [44].

A realistic model applicable to the experiment should include loss terms, the main source of which are threebody collisions in the bosonic condensate. In fact, the losses were analyzed in detail, in the present contexts, in Refs. [3] and [19] (including supplemental materials of both publications). It was demonstrated, theoretically and experimentally, that the losses, although they may be conspicuous, allow one to work with solitons for quite a long time, which is completely sufficient for the creation and observation of the QDs.

The total norm of the wave function, which is a dynamical invariant of the model, being proportional to the total number of atoms in the dual-core system, is

$$
N=N_{1}+N_{2} \equiv \int_{-\infty}^{+\infty} d x\left(\left|\Psi_{1}\right|^{2}+\left|\Psi_{2}\right|^{2}\right) \text {. }
$$

Also conserved are the system's Hamiltonian and total momentum:

$$
\begin{gathered}
H=\int_{-\infty}^{+\infty} d x\left[\sum_{n=1,2}\left(\frac{1}{2}\left|\partial_{x}\left(\Psi_{n}\right)\right|^{2}+\frac{1}{2}\left|\Psi_{n}\right|^{4}-\frac{2}{3}\left|\Psi_{n}\right|^{3}\right)\right. \\
\left.-\kappa\left(\Psi_{1} \Psi_{2}^{*}+\text { c.c. }\right)\right] \\
P=i \int_{-\infty}^{+\infty} d x \sum_{n=1,2} \Psi_{n} \partial_{x}\left(\Psi_{n}^{*}\right)
\end{gathered}
$$

where both $*$ and c.c. stand for the complex conjugation.

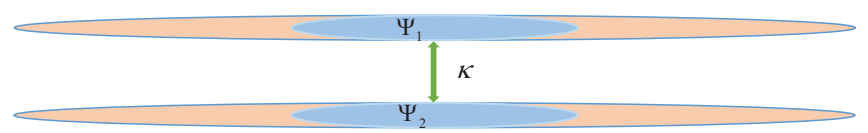

FIG. 1: (Color online) The schematic of the system: quantum droplets (denoted by the blue color), which are formed in the Bose gas, are trapped in the symmetric dual-core quasi-onedimensional potential (the orange areas remain empty). The parallel cores are coupled by hopping with rate $\kappa$.

Stationary QDs with chemical potential $\mu$ are sought for as a solution to Eq. (11) in the form of

$$
\left\{\Psi_{1}, \Psi_{2}\right\}=\left\{\psi_{1}, \psi_{2}\right\} e^{-i \mu t}
$$

with real stationary wave functions $\psi_{1}$ and $\psi_{2}$ obeying equations (the prime stands for $d / d x$ )

$$
\begin{aligned}
& \mu \psi_{1}=-\frac{1}{2} \psi_{1}^{\prime \prime}+\psi_{1}^{3}-\psi_{1}^{2}-\kappa \psi_{2}, \\
& \mu \psi_{2}=-\frac{1}{2} \psi_{2}^{\prime \prime}+\psi_{2}^{3}-\psi_{2}^{2}-\kappa \psi_{1} .
\end{aligned}
$$

Symmetric QD solutions of Eq. (5), with $\psi_{1}=\psi_{2}$ and the chemical potential taking values

$$
-2 / 9<\mu+\kappa<0
$$

have the known form [18]:

$$
\begin{gathered}
\psi_{1,2}=\frac{-3(\mu+\kappa)}{1+\sqrt{1+(9 / 2)(\mu+\kappa)} \cosh (\sqrt{-2(\mu+\kappa)} x)} \\
\equiv \psi_{\text {symm }}(x) .
\end{gathered}
$$

In the limit of $(\mu+\kappa) \rightarrow-0$, they take the bell-shaped form,

$$
\psi_{1,2} \approx \frac{-3(\mu+\kappa)}{2 \cosh ^{2}(\sqrt{-(\mu+\kappa) / 2} x)} .
$$

In the opposite limit of

$$
\mu+\kappa \rightarrow-2 / 9
$$


[see Eq. (6)], the soliton features an extended flat-top shape, with a nearly constant intrinsic wave function,

$$
\psi_{1,2} \approx 2 / 3
$$

of size

$$
L \approx(3 / 2) \ln \left((\mu+\kappa+9 / 2)^{-1}\right)
$$

This flat-top wave form is bounded by two fronts, which are represented by exact solutions of Eq. (5), available precisely at $\mu+\kappa=-2 / 9$ :

$$
\psi_{1,2}=\frac{2 / 3}{1+\exp \left[ \pm(2 / 3)\left(x-x_{0}\right)\right]},
$$

( $x_{0}$ is an arbitrary shift of the coordinate), each interpolating between $\psi_{1,2}=0$ and $\psi_{1,2}=2 / 3$, cf. Eq. (10). The energy of the front pattern, calculated as per Eq. (2), is

$$
H_{\text {front }}=8 / 81 \text {. }
$$

A similar exact front solution of the GPE with the cubicquintic nonlinearity is known too [58].

The SSB point is determined by the condition that the linearization of Eq. (5) around the symmetric soliton produces a critical antisymmetric eigenmode with the zero eigenvalue, $\delta \psi_{1,2}= \pm \delta \psi_{0}$, which satisfies the linear equation [38, 50],

$$
(\mu-\kappa) \delta \psi_{0}=\left[-\frac{1}{2} \frac{d^{2}}{d x^{2}}+3 \psi_{\mathrm{symm}}^{2}(x)-2 \psi_{\mathrm{symm}}(x)\right] \delta \psi_{0} .
$$

In this work, we have obtained numerical asymmetric solutions of Eq. (5) by means of the finite-difference method. Eq. (14) it is used to predict the SSB point in an analytical approximation, see Eq. (20) below.

It is relevant to stress that all the modes which are antisymmetric or asymmetric with respect to the parallelcoupled cores are spatial even ones, i.e., $\psi_{1,2}(-x)=$ $\psi_{1,2}(x)$. On the other hand, it follows from Eq. (5) that Eq. (14) with $\kappa=0$ has an obvious exact solution, which, however, is spatially odd,

$$
\delta \psi_{0}(x ; \kappa=0)=\frac{\partial}{\partial x}\left[\psi_{\text {symm }}(x ; \kappa=0)\right]
$$

This fact implies that solutions asymmetric with respect to the two cores cannot branch off from the symmetric ones at $\kappa=0$, keeping the spatial parity.

The linear-stability analysis for the stationary states was performed by adding small perturbations to solution (4):

$$
\begin{aligned}
& \Psi_{1}(x, t)=\left[\psi_{1}+\varepsilon w_{1} e^{i G t}+\varepsilon v_{1}^{*} e^{-i G^{*} t}\right] e^{-i \mu t}, \\
& \Psi_{2}(x, t)=\left[\psi_{2}+\varepsilon w_{2} e^{i G t}+\varepsilon v_{2}^{*} e^{-i G^{*} t}\right] e^{-i \mu t}
\end{aligned}
$$

where $\varepsilon$ is a real infinitesimal amplitude of the perturbation with eigenfunctions $w_{1}, w_{2}, v_{1}$ and $v_{2}$. The substitution of expression (16) in Eq. (1) and subsequent linearization leads to the eigenvalue problem in the matrix form,

$$
\left(\begin{array}{cccc}
\hat{L}_{1} & -\kappa & \hat{L}_{3} & 0 \\
-\kappa & \hat{L}_{2} & 0 & \hat{L}_{4} \\
-\hat{L}_{3}^{*} & 0 & -\hat{L}_{1} & \kappa \\
0 & -\hat{L}_{4}^{*} & \kappa & -\hat{L}_{2}
\end{array}\right)\left(\begin{array}{c}
w_{1} \\
w_{2} \\
v_{1} \\
v_{2}
\end{array}\right)=-G\left(\begin{array}{c}
w_{1} \\
w_{2} \\
v_{1} \\
v_{2}
\end{array}\right)
$$

with operators

$$
\begin{gathered}
\hat{L}_{1}=-\frac{1}{2} \partial_{x x}-\mu+2\left|\psi_{1}\right|^{2}-\frac{3}{2}\left|\psi_{1}\right|, \\
\hat{L}_{2}=-\frac{1}{2} \partial_{x x}-\mu+2\left|\psi_{2}\right|^{2}-\frac{3}{2}\left|\psi_{2}\right|, \\
\hat{L}_{3}=\psi_{1}^{2}-\frac{\psi_{1}^{2}}{2\left|\psi_{1}\right|}, \\
\hat{L}_{4}=\psi_{2}^{2}-\frac{\psi_{2}^{2}}{2\left|\psi_{2}\right|} .
\end{gathered}
$$

The linear eigenvalue problem based on Eq. (17) can be solved by means of the finite- difference method. As usual, the existence of an imaginary part in a perturbation eigenfrequency, $G$, implies an instability.

\section{SYMMETRIC AND ASYMMETRIC QUANTUM DROPLETS}

\section{A. Generic numerical results}

Solutions for QDs which are symmetric and asymmetric with respect to the coupled symmetric cores were produced with the help of the imaginary-time-integration method [59, 60], applied to Eq. (11). Figure 2 displays typical examples of stable and unstable QDs with different values of norm $N$. Similar to the situation in the single-core model [see Eqs. (8) and (11)], QDs in the present system feature spatial density profiles of two different types: bell-shaped [see Figs. 2(a1)-(c1), (e1)] and flat-top ones [Figs. 2(d1)], for relatively small and large values of $N$, respectively.

Examples of stable symmetric and asymmetric QDs can be seen, respectively, in Figs. 2(a1), (b1), (d1) and (c1), (e1). The asymmetry is characterized by parameter

$$
\delta \equiv\left|\frac{N_{1}-N_{2}}{N_{1}+N_{2}}\right| .
$$

Accordingly, the SSB is characterized by dependences of $\delta$ on $N$ and $\kappa$.

First, in Fig. 3 we produce $\delta(N)$ curves for different values of $\kappa$. Due to the competition between the quadratic self-attraction and cubic repulsion, they take the form of bifurcation loops (somewhat similar to those in the cubic-quintic model [44]), which exist at 

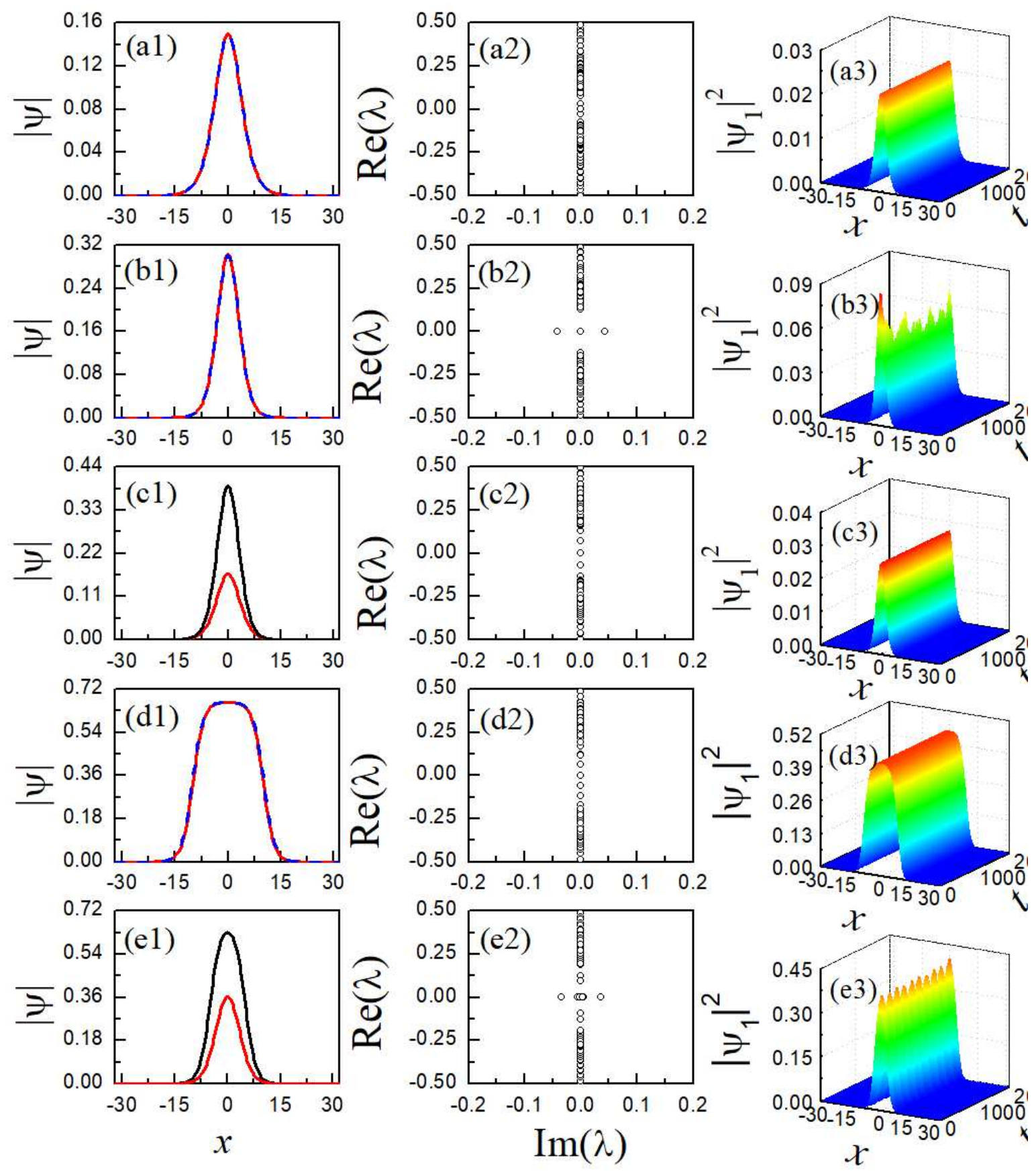

FIG. 2: (Color online) Profiles of $\left|\psi_{1}(x)\right|$ and $\left|\psi_{2}(x)\right|$ components of the QDs, and the exact solution given by Eq. (7), are shown by solid black, dotted red, and dashed gray curves, respectively, for different values of the total norm, $N=0.3,1,1$, 20, and 4, severally, in panels (a1)-(e1). These examples of self-trapped modes correspond to points a, b, c, and e, which are marked in Figs. 3(a,c) [point d is not marked, as the respective value of the norm, $N=20$, is located beyond the frame of Fig. 3(c)]. Perturbation eigenvalues for the corresponding symmetric [in (a1,b1,d1)] and asymmetric [in (c1,e1)] QDs, and direct simulations of the perturbed evolution of their $\Psi_{1}$ component, are displayed, respectively, in panels (a2)-(e2) and (a3)-(e3). Parameters of Eq. (1) are $\kappa=0.05$ in panels (a3)-(d3) and $\kappa=0.03$ in panels (e3); the amplitude of small random perturbations in Eq. (16) is $\varepsilon=0.01$.

$\kappa \leq \kappa_{\max } \approx 0.0592$. With the increase of $N$, the $\delta(N)$ curves first show the SSB bifurcation of the supercritical (forward) type, driven by the quadratic self-attraction, which is followed by a reverse symmetry-restoring bifur- cation, which occurs when the cubic repulsion becomes a dominant nonlinear term. The latter bifurcation is of the subcritical (backward) type, which lends the loop a concave shape, at $\kappa<\kappa_{0} \approx 0.044<\kappa_{\max }$. In the interval 
of $\kappa_{0}<\kappa<\kappa_{\max }$, the symmetry-restoring bifurcation is supercritical, making the loop a convex figure, which shrinks at $\kappa \rightarrow \kappa_{\max }$ and disappears at $\kappa=\kappa_{\max }$. It is relevant to mention that the bifurcations of the subcritical and supercritical types are tantamount to phase transitions of the the first and second kinds, respectively (see, e.g., Ref. [45] and references therein), thus predicting the possibilities of these phase transition in the QDs trapped in the dual-core potential.
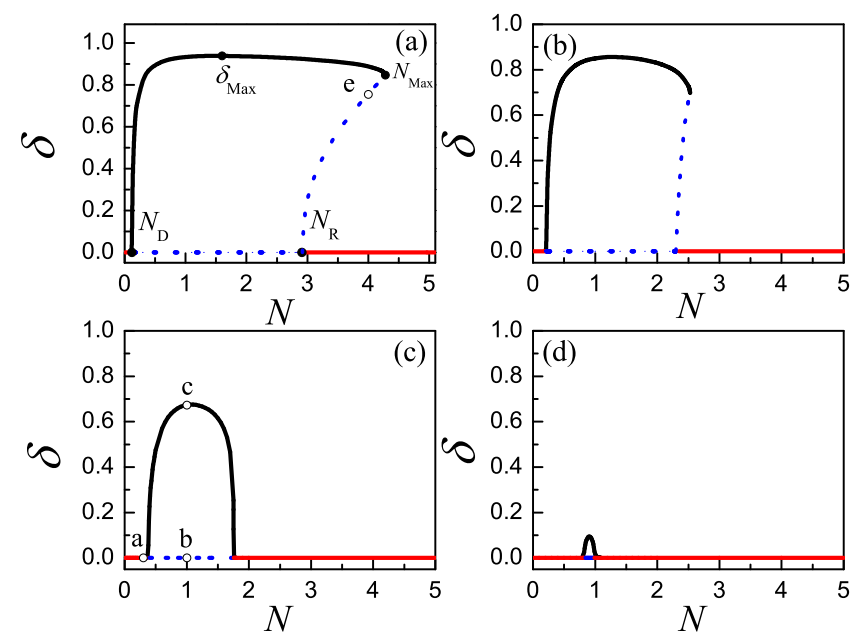

FIG. 3: (Color online) A set of bifurcation diagrams for symmetric and asymmetric QDs, in the plane of $(N, \delta)$, as found from numerical solution of Eqs. (5) at different values of the linear-coupling parameter, $\kappa$ : (a) $\kappa=0.03$; (b) $\kappa=0.04$; (c) $\kappa=0.05$; (d) $\kappa=0.059$. Red, dotted blue, and black curves represent symmetric stable, symmetric unstable, and asymmetric stable states, respectively.

The bifurcation loops are chiefly built of the QDs of the bell-shaped (sech) type, corresponding to relatively small and moderate values of the norm, while the flat-top modes are found for large values of $N$, at which the SSB is, in most cases, suppressed by the strong self-repulsive nonlinearity.

Results pertaining to the bifurcation loops are collected in Fig. 4. Namely, Fig. 4(a) displays values $N_{\mathrm{D}}$ and $N_{\mathrm{R}}$ of the total norm at the direct- and reversebifurcation points, which merge at $\kappa=\kappa_{\max }$, and Fig. 4(b) shows the largest value of asymmetry (19), $\delta_{\max }(\kappa)$, as a function of the coupling constant. Figure 4(a) also includes a plot (the blue line) showing the largest value $N_{\text {max }}$ of $N$ attained in the concave loops, in the case of $\kappa<\kappa_{0}$. Obviously, $N_{\max } \equiv N_{\mathrm{R}}$ at $\kappa_{0}<\kappa<\kappa_{\max }$.

\section{B. Analytical results for the weakly-coupled system}

Approximate analytical results can be obtained in the limit of small $\kappa$ and, accordingly, small $N_{\mathrm{D}}$. In this case, Eq. (14) with approximation (8) adopted for $\psi_{\text {symm }}$, can be solved exactly, using the well-known result from quantum mechanics, similar to how this was done, in another
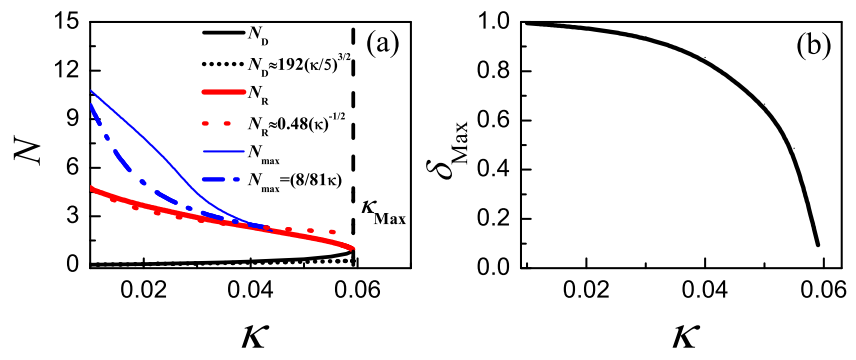

FIG. 4: (Color online) (a) Values of the norm of the symmetric solution at which the direct and reverse bifurcations occur $\left(N_{\mathrm{D}}\right.$ and $N_{\mathrm{R}}$, respectively). The two curves merge and terminate at $\kappa=\kappa_{\max } \approx 0.0592$. The blue line shows the largest value of the norm, $N_{\max }$, attained by asymmetric solitons, at which the stable and unstable branches meet in the concave bifurcation loop, see Figs. 3(a,b). $N_{\max }$ merges with $N_{\mathrm{R}}$ at $\kappa=\kappa_{0} \approx 0.044$, the loops being convex at $\kappa>\kappa_{0}$. The dotted black line, short-dashed red line, and dashed-dotted blue line show analytical approximation (20), (25) and (24) for $N_{\mathrm{D}}, N_{\mathrm{R}}$, and $N_{\max }$, respectively (coefficient 0.48 in the analytical expression for $N_{\mathrm{R}}$ is a fitting constant). (b) The maximum values of the asymmetry parameter, $\delta$, of the solutions generated by the bifurcation [see Eq. [19]), versus the linear-coupling parameter $\kappa$.

context (dual-core optical fibers), in Refs. [38, 50]:

$$
\mu \approx-(13 / 5) \kappa, N_{\mathrm{D}} \approx 192(\kappa / 5)^{3 / 2}
$$

In Fig. 4(a), the approximate dependence given by the second equality in Eq. (20), is plotted by the dotted black line, showing that it fits well to the numerically found dependence at $\kappa \lesssim 0.04$.

Further, both $N_{\max }$ and $N_{\mathrm{R}}$ diverge in the limit of $\kappa \rightarrow 0$, when one component in the asymmetric state (e.g., $\left.\psi_{2}\right)$ is vanishing, its amplitude in the flat-top states (which correspond to large $N$ ) being

$$
\psi_{2} \approx 3 \kappa
$$

as it follows from Eqs. (5), (9), and (10), while a correction to the amplitude of the larger component is determined by the conservation of the total norm:

$$
\psi_{1} \approx 2 / 3-(27 / 4) \kappa^{2} \text {. }
$$

At $\kappa \rightarrow 0$, the value of $N_{\max }$ can be estimated, taking into regard that the energy of the flat-top symmetric soliton is larger than its single-component counterpart, with the same total norm, by the amount equal to the front's energy (13), as the single-component state includes only two fronts, unlike four ones in the two-component state, and the energy (13) actually pertains to the double front in the two-component symmetric state. Effectively, this is an energy barrier which maintains the existence of the asymmetric soliton. On the other hand, the weak linear coupling between the components in the flat-top symmetric state of length $L$ corresponds to the negative energy, which is

$$
H_{\text {coupling }} \approx-(8 / 9) \kappa L \text {, }
$$




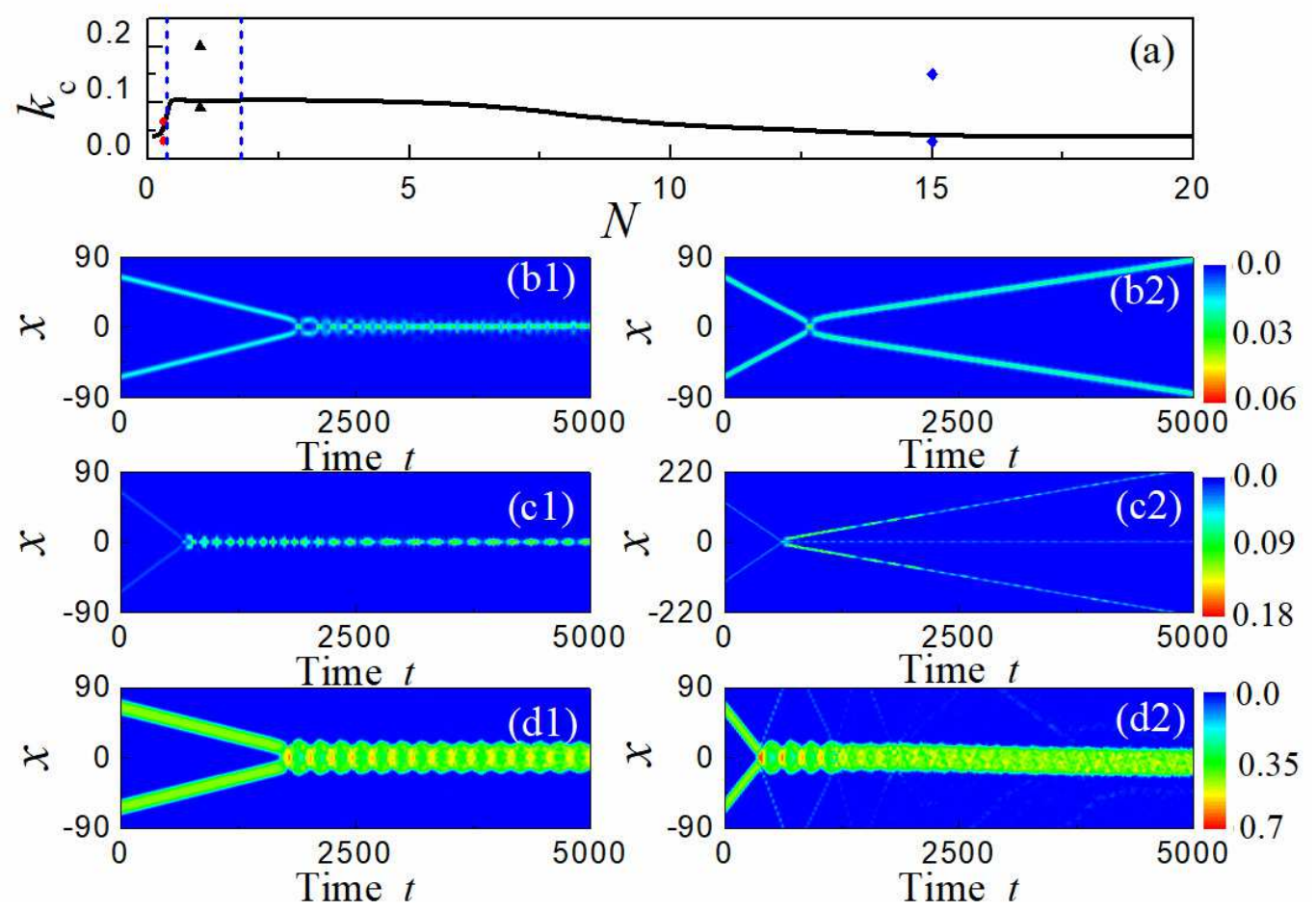

FIG. 5: (Color online) (a) The value of the kick, $k_{c}$, which is a boundary between the merger and passage of colliding QDs, launched as per Eq. (26) with $D=64$, versus the total norm, $N$. Typical examples of the density plots of the colliding droplets: (b1) $N=0.3, k=0.03 ;$ (b2) $N=0.3, k=0.065 ;$ (c1) $N=1, k=0.09 ;$ (c2) $N=1, k=0.2 ;(\mathrm{d} 1) N=15, k=0.03 ;(\mathrm{d} 2)$ $N=15, k=0.15$. In this figure, $\kappa=0.05$ is fixed. The colliding QDs are symmetric in (b1,b2) and asymmetric in (c1,c2).

according to Eq. (2). The asymmetric state ceases to exist, by a jump [like in Fig. 3(a)] under condition $H_{\text {front }}+H_{\text {coupling }}<0$, i.e., at $L>L_{\max } \approx(9 \kappa)^{-1}$. Eventually, the respective prediction for the largest norm, up to which the asymmetric states exist at $\kappa \rightarrow 0$, is

$$
N_{\max } \approx 2(2 / 3)^{2} L_{\max }=8 /(81 \kappa) .
$$

Further, in the same limit of $\kappa \rightarrow 0$, value $N_{\mathrm{R}}$ at the reverse-bifurcation point also diverges, because, as shown above [see Eq. (15)], the SSB cannot take place in the form of an asymmetric branch stemming from a symmetric one at $\kappa=0$ and some finite value of $N$. A rough estimate for the divergence can be derived by noting that large size $L$ of the symmetric QD is associated with a shift of the eigenvalue in Eq. (14), $\kappa \sim L^{-2}$, hence the respective norm is estimated as

$$
N_{\mathrm{R}} \approx 2(3 / 2)^{2} L \sim \kappa^{-1 / 2},
$$

cf. Eq. (24).

The dotted black line, dash-dotted blue line, and shortdashed red line show the analytical approximations given by Eqs. (20), (24) and (25) for $N_{\mathrm{D}}, N_{\max }$, and $N_{\mathrm{R}}$, respectively (the curve representing $N_{\mathrm{R}}$ is drawn with a fitting parameter). It is seen that the analytically predicted values $N_{\mathrm{D}}$ and $N_{\mathrm{R}}$ agree well with their numerical counterparts. The prediction given by Eq. (24) is less ac- curate, in comparison with the numerical findings, but, still, it is qualitatively correct.

\section{COLLISIONS OF TWO-COMPONENT DROPLETS}

Once stable solitons are available in the Galileaninvariant system (10), it is relevant to explore collisions between them. In the framework of the usual dual-core system with the cubic self-attractive nonlinearity, collisions were studied in Ref. 61], demonstrating, chiefly, merger of the colliding solitons into strongly oscillating breathers, unless the solitons would pass through each other if the collision velocity was very large.

We simulated the collisions, solving Eq. (1) with initial conditions

$$
\Psi_{1,2}(x, t=0)=\psi_{1,2}(x+D) e^{i k x}+\psi_{1,2}(x-D) e^{-i k x+\varphi},
$$

where $\psi_{1,2}$ represent the stationary shape of twocomponent QDs, $\pm k$ is a kick, which sets two initial droplets, separated by distance $2 D$, in motion with velocities also equal to $\pm k$, and $\varphi$ is the initial phase difference between them.

The simulations demonstrate a trend to inelastic outcomes of the collisions between the solitons in the inphase configuration, i.e., with $\varphi=0$ in Eq. (26). QDs 

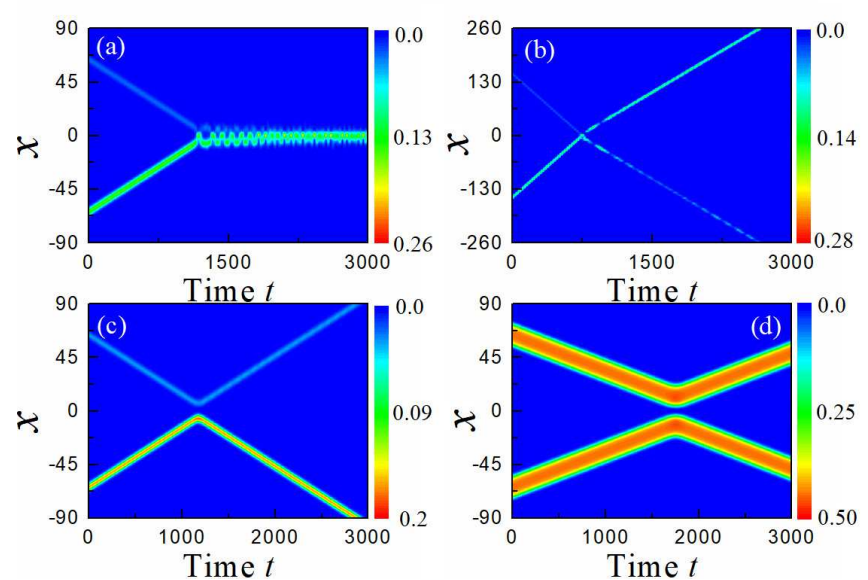

FIG. 6: (Color online) Typical examples of density plots, in component $\Psi_{1}$, for collisions between two asymmetric QDs with opposite placement of the larger and smaller components with respect to the two cores. Panels (a) and (b) display, severally, strongly inelastic and quasi-elastic collisions between in-phase solitons with norms $N=1$, the respective kicks being $k_{\mathrm{a}}=0.05$ and $k_{\mathrm{b}}=0.20$. Panels (c) and (d) show completely elastic collisions between out-of-phase QDs, i.e., ones with $\varphi=\pi$ in Eq. (26), for $N=1, k=0.05$ and $N=15, k=0.03$, respectively. In this figure, $\kappa=0.05$ is fixed.

of the bell (sech)-shaped type merge at relatively small values of $k$, and collide quasi-elastically (passing through each other) at large $k$. A boundary value, $k_{c}$, which separates the inelastic and elastic collisions is displayed in Fig. 5 (a), as a function of $N$, for $\kappa=0.05$. In particular, it demonstrates that $k_{c}$ is smaller for symmetric bell-shaped QDs than for asymmetric ones of the same type. This difference is explained by the fact that the nonlinear interaction between larger components in the asymmetric state is stronger than in the symmetric one, hence larger kinetic energy is necessary to overcome the interaction and let the colliding QDs pass through each other.

Figures $5(\mathrm{~b} 1, \mathrm{c} 1)$ and $(\mathrm{b} 2, \mathrm{c} 2)$ show typical collision pictures for $k<k_{c}$ and $k>k_{c}$, respectively. The examples displayed in panels $(\mathrm{b} 1, \mathrm{~b} 2)$ and $(\mathrm{c} 1, \mathrm{c} 2)$ correspond, respectively, to the red dot and black triangle marks in panel (a). These pictures demonstrate that, when the bell-shaped QDs pass through each other at $k>k_{c}$, the collisions essentially perturb them. In particular, symmetric QDs emerge from the collision with excited intrinsic oscillations and velocities different from the original ones. In addition, colliding asymmetric bell-shaped QDs generate an extra oscillating localized pulse (breather) with zero velocity.

We have also considered cross-symmetric collisions between two asymmetric QDs, i.e., with opposite placements of the larger and smaller components with respect to the two cores, as shown in Fig. 6(a)-(b). In this case, strongly inelastic, quasi-elastic, and completely elastic outcomes are observed too.

As the bell-shaped QDs carry over into flat-top ones with the increase of $N$, the newly generated quiescent breather grows larger, and eventually absorbs almost all the norm of the colliding QDs, see an example in Fig. 5(d2) (a similar outcome of collisions of single-component QDs was reported in Ref. [18]). Actually, this is a different mechanism of the merger of colliding QDs, cf. panels (d1) and (d2) in Fig. 55, which correspond to the blue rhombic marks in Fig. 5(a). We find that about $91 \%$ of the total norm is absorbed by the quiescent breather in Fig. 5(d2).

Lastly, also similar to the results reported in Ref. [18] for the single-component model, completely elastic collisions (rebounds) occur between the two-component QDs with opposite signs, i.e., $\varphi=\pi$ in Eq. (26), as shown in 6 $(\mathrm{c}, \mathrm{d})$, and is observed in other cases too.

\section{CONCLUSION}

The objective of this work is to study the SSB (spontaneous symmetry breaking) of effectively one-dimensional QDs (quantum droplets) created in the binary bosonic gas loaded in the dual-core trapping potential. The matter-wave dynamics in this system is governed by the linearly-coupled GPEs (Gross-Pitaevskii equations) with the cubic repulsive and quadratic attractive nonlinear terms, the latter ones being represented by the LHY (Lee-Huang-Yang) correction to the mean-field approximation. QDs in this system feature bell (sech)-shaped density profiles for smaller values of total norm, $N$, and flat-top profiles for larger $N$. The SSB bifurcation takes place with the increase of $N$, while the QDs keep the sech shape. Further increase of $N$ leads to the restoration of the symmetry via the reverse bifurcation, hence the flattop QDs, which realize large values of $N$, are symmetric, in most cases. The resulting bifurcations loops are concave and convex in the cases of small and larger values of the inter-core coupling constant, and vanish when it exceeds a critical value. Some results have been obtained in the analytical form - in particular, the exact solution was produced for a front separating zero and finite constant values of the wave function, in the flat-top states.

Collisions between two-component QDs have been considered too. Unless the colliding in-phase QDs move very fast, they tend to demonstrate inelastic interactions, leading to their merger into breathers.

An interesting extension of the present analysis is to perform it for a two-dimensional dual-core system, where the effective nonlinear terms in the GPE is different, $\sim|\Psi|^{2} \Psi \ln \left(|\Psi|^{2}\right)[5$, 35, 36]. In that case, it will be possible to study the SSB not only in fundamental twocomponent QDs, but also in ones with embedded vorticity, cf. Refs. [53, 62]. 


\section{Acknowledgments}

This work was supported by NNSFC (China) through grants No. 11874112, 11575063, 11547007, and by the
Israel Science Foundation through grant No. 1287/17.
[1] M. Schmitt, M. Wenzel, F. Böttcher, I. Ferrier-Barbut, and T. Pfau, Self-bound droplets of a dilute magnetic quantum liquid, Nature 539, 259 (2016).

[2] L. Chomaz, S. Baier, D. Petter, M. J. Mark, F. Wächtler, L. Santos, and F. Ferlaino, Quantum-fluctuation-driven crossover from a dilute Bose-Einstein condensate to a macrodroplet in a dipolar quantum fluid, Phys. Rev. X 6, 041039 (2016).

[3] C. R. Cabrera, L. Tanzi, J. Sanz, B. Naylor, P. Thomas, P. Cheiney, L. Tarruell, Quantum liquid droplets in a mixture of Bose-Einstein condensates, Science 359, 301 (2018).

[4] D. S. Petrov, Quantum mechanical stabilization of a collapsing Bose-Bose mixture, Phys. Rev. Lett. 115, 155302 (2015).

[5] D. S. Petrov and G. E. Astrakharchik, Ultradilute lowdimensional liquids, Phys. Rev. Lett. 117, 100401 (2016).

[6] T. D. Lee, K. S. Huang, and C. N. Yang, Eigenvalues and eigenfunctions of a Bose system of hard spheres and Its Low-temperature properties, Phys. Rev. 106, 1135 (1957).

[7] F. Wächtler and L. Santos, Quantum filaments in dipolar Bose-Einstein condensates, Phys. Rev. A 93, 061603(R) (2016).

[8] F. Wächtler and L. Santos, Ground-state properties and elementary excitations of quantum droplets in dipolar Bose-Einstein condensates, Phys. Rev. A 94, 043618 (2016).

[9] D. Baillie, R. M. Wilson, R. N. Bisset, and P. B. Blakie, Self-bound dipolar droplet: A localized matter wave in free space, Phys. Rev. A 94, 021602(R) (2016).

[10] D. Edler, C. Mishra, F. Wähtler, R. Nath, S. Sinha, and L. Santos, Quantum Fluctuations in Quasi-OneDimensional Dipolar Bose-Einstein Condensates, Phys. Rev. Lett. 119, 050403 (2017).

[11] I. Ferrier-Barbut, H. Kadau, M. Schmitt, M. Wenzel, and T. Pfau, Observation of Quantum Droplets in a Strongly Dipolar Bose Gas, Phys. Rev. Lett. 116, 215301 (2016).

[12] I. Ferrier-Barbut,. M. Wenze, F. Bötcher, T. Langen, M. Isoard, S. Stringari, and T. Pfau, Scissors Mode of Dipolar Quantum Droplets of Dysprosium Atoms, Phys. Rev. Lett. 120, 160402 (2018).

[13] A. Cidrim, F. E. A. dos Santos, E. A. L. Henn, and T. Macrí, Vortices in self-bound dipolar droplets, Phys. Rev. A 98, 023618 (2018).

[14] R. N. Bisset, R. M. Wilson, D. Baillie, and P. B. Blakie, Ground-state phase diagram of a dipolar condensate with quantum fluctuations, Phys. Rev. A. 94, 033619 (2016).

[15] Y. Sekino and Y. Nishida, Quantum droplet of onedimensional bosons with a three-body attraction, Phys. Rev. A 97, 011602(R) (2018).

[16] C. Staudinger, F. Mazzanti, and R. E. Zillich, Self-bound Bose mixtures, Phys. Rev. A 98, 023633 (2018).

[17] V. Cikojević, K. Dželalija, P. Stipanović, and L. V. Markić, Ultradilute quantum liquid drops, Phys. Rev.
B 97, 140502 (R) (2018).

[18] G. E. Astrakharchik and B. A. Malomed, Dynamics of one-dimensional quantum droplets, Phys. Rev. A 98, 013631 (2018).

[19] P. Cheiney, C. R. Cabrera, J. Sanz, B. Naylor, L. Tanzi, and L. Tarruell, Bright soliton to quantum droplet transition in a mixture of Bose-Einstein condensates, Phys. Rev. Lett. 120, 135301 (2018).

[20] G. Semeghini, G. Ferioli, L. Masi, C. Mazzinghi, L. Wolswijk, F. Minardi, M. Modugno, G. Modugno, M. Inguscio, and M. Fattori, Self-bound quantum droplets in atomic mixtures, Phys. Rev. Lett. 120, 235301 (2018).

[21] A. Cappellaro, T. Macrì, and L. Salasnich, Collective modes across the soliton-droplet crossover in binary Bose mixtures, Phys. Rev. A 97, 053623 (2018).

[22] A. Pricoupenko, and D. S. Petrov, Dimer-dimer zero crossing and dilute dimerized liquid in a one-dimensional mixture, Phys. Rev. A 97, 063616 (2018).

[23] A. Cappellaro, T. Macrí, G. F. Bertacco, and L. Salasnich, Equation of state and self-bound droplet in Rabicoupled Bose mixtures, Sci. Rep. 7, 13358 (2017).

[24] X. Cui, Spin-orbit-coupling-induced quantum droplet in ultracold Bose-Fermi mixtures, Phys. Rev. A. 98, 023630 (2018).

[25] N. Westerberg, K. E. Wilson, C. W. Duncan, D. Faccio, E. M. Wright, P. Öhberg, and M. Valiente, Self-bound droplets of light with orbital angular momentum, Phys. Rev. A. 98, 053835 (2018).

[26] B. Laburthe-Tolra, A strange kind of liquid, Nature 539, 176 (2016).

[27] B. A. Malomed, D. Mihalache, F. Wise, and L. Torner. Spatiotemporal optical solitons, J. Optics B: Quant. Semicl. Opt. 7, R53 (2005).

[28] B. A. Malomed, Multidimensional solitons: Wellestablished results and novel findings, Eur. Phys. J. Special Topics 225, 2507 (2016).

[29] H. Sakaguchi, B. Li, and B. A. Malomed, Creation of two-dimensional composite solitons in spin-orbit-coupled self-attractive Bose-Einstein condensates in free space, Phys. Rev. E 89, 032920 (2014).

[30] Y.-C. Zhang, Z.-W. Zhou, B. A. Malomed, and H. Pu, Stable solitons in three dimensional free space without the ground state: Self-trapped Bose-Einstein condensates with spin-orbit coupling, Phys. Rev. Lett. 115, 253902 (2015).

[31] C. Lee, J. Huang, H. Deng, H. Dai, and J. Xu, Nonlinear quantum interferometry with Bose condensed atoms, Front. Phys. 7, 1090130 (2012).

[32] A. D. Martin and J. Ruostekoski, Quantum dynamics of atomic bright solitons under splitting and recollision, and implications for interferometry, New J. Phys. 14, 043040 (2012).

[33] G. D. McDonald, C. C. N. Kuhn, K. S. Hardman, S. Bennetts, P. J. Everitt, P. A. Atlin, J. E. Debs, J. D. Close, and N. P. Robins, Bright solitonic matter-wave 
interferometer, Phys. Rev. Lett. 113, 013002 (2014).

[34] R. K. Bullough and M. Wadati, Optical solitons and quantum solitons, J. Opt. B: Quant. Semicl. Opt. 6, S205 (2004).

[35] Y. Li, Z. Chen, Z. Luo, C. Huang, H. Tan, W. Pang, and B. A. Malomed, Two-dimensional vortex quantum droplets, Phys. Rev. A 98, 063602 (2018).

[36] Y. Li, Z. Luo, Y. Liu, Z. Chen, C. Huang, S. Fu, H. Tan, and B. A. Malomed, Two-dimensional solitons and quantum droplets supported by competing self- and crossinteractions in spin-orbit-coupled condensates, New J. Phys. 19, 113043(2017).

[37] Y. V. Kartashov, B. A. Malomed, L. Tarruell, and L. Torner, Three-dimensional droplets of swirling superfluids, Phys. Rev. A 98, 013612 (2018).

[38] E. M. Wright, G. I. Stegeman, and S. Wabnitz, Solitarywave decay and symmetry-breaking instabilities in twomode fibers, Phys. Rev. A 40, 4455 (1989).

[39] A. W. Snyder, D. J. Mitchell, L. Poladian, D. R. Rowland, and Y. Chen, Physics of nonlinear fiber couplers, J. Opt. Soc. Am. B 8, 2102 (1991).

[40] A. I. Maimistov, Propagation of a light pulse in nonlinear tunnel-coupled optical waveguides. Kvant. Elektron. 18, 758-761 [Sov. J. Quantum Electron. 21, 687 (1991)].

[41] S. Trillo, S. Wabnitz, E. M. Wright, and G. I. Stegeman, Soliton switching in fiber nonlinear directional couplers, Opt. Lett. 13, 672 (1998).

[42] P. G. Kevrekidis, Z. Chen, B. A. Malomed, D. J. Frantzeskakis, and M. I. Weinstein, Spontaneous symmetry breaking in photonic lattices: theory and experiment. Phys. Lett. A 340, 275 (2005).

[43] G. Herring, P. G. Kevrekidis, B. A. Malomed, R. Carretero-González, and D. J. Frantzeskakis, Symmetry breaking in linearly coupled dynamical lattices, Phys. Rev. E 76, 066606 (2007).

[44] L. Albuch, B. A. Malomed, Transitions between symmetric and asymmetric solitons in dual-core systems with cubic-quintic nonlinearity, Math. Comput. Simulat. 74, 312 (2007).

[45] Z. Mai, W. Pang, J. Wu, and Y. Li, Symmetry Breaking of discrete solitons in two-component waveguide arrays with long-range linearly coupled effect, J. Phys. Soc. Jpn. 84, 014401 (2015).

[46] T. Heil, I. Fischer,W. Elsässer, J. Mulet, C. R. Mirasso, Chaos synchronization and spontaneous symmetrybreaking in symmetrically delay-coupled semiconductor lasers. Phys. Rev. Lett. 86, 795 (2000).

[47] P. Hamel, S. Haddadi, F. Raineri, P. Monnier, G. Beaudoin, I. Sagnes, A. Levenson, and A. M. Yacomotti, Spontaneous mirror-symmetry breaking in coupled photoniccrystal nanolasers, Nat. Photonics 9, 311-315 (2015).

[48] M. Liu, D. A. Powell, I. V. Shadrivov, M. Lapine, and Y. S. Kivshar, Spontaneous chiral symmetry breaking in metamaterials, Nat. Commun. 5, 4441 (2014).
[49] Spontaneous Symmetry Breaking, Self-Trapping, and Josephson Oscillations, ed. by B. A. Malomed (Springer, Berlin, 2013)

[50] B. A. Malomed, Spontaneous symmetry breaking in nonlinear systems: An overview and a simple model, in: Nonlinear Dynamics: Materials, Theory and Experiments, ed. by M. Tlidi and M. G. Clerc (Springer: Heidelberg, 2016).

[51] G. J. Milburn, J. Corney, E. M. Wright, and D. F. Walls, Quantum dynamics of an atomic Bose- Einstein condensate in a double-well potential, Phys. Rev. A 55, 4318 (1997).

[52] A. Smerzi, S. Fantoni, S.Giovanazzi, and S. R. Shenoy, Quantum coherent atomic tunneling between two trapped Bose-Einstein condensates, Phys. Rev. Lett. 79, 4950 (1997).

[53] A. Gubeskys and B. A. Malomed, Symmetric and asymmetric solitons in linearly coupled Bose-Einstein condensates trapped in optical lattices, Phys. Rev. A 75, 063602 (2007).

[54] M. Matuszewski, B. A. Malomed, and M. Trippenbach, Spontaneous symmetry breaking of solitons trapped in a double-channel potential, Phys. Rev. A 75, 063621 (2007).

[55] Y. Li, J. Liu, W. Pang, and B. A. Malomed, Symmetry breaking in dipolar matter-wave solitons in dual-core couplers, Phys. Rev. A 87, 013604 (2013).

[56] Y. Li, Z. Fan, Z. Luo, Y. Liu, H. He, J. Lü, J. Xie, C. Huang, H. Tan, Cross-symmetry breaking of twocomponent discrete dipolar matter-wave solitons, Front. Phys. 12, 124206 (2017).

[57] Z. Chen, Y. Li, B. A. Malomed, and L. Salasnich, Spontaneous symmetry breaking of fundamental states, vortices, and dipoles in two- and one-dimensional linearly coupled traps with cubic self-attraction, Phys. Rev. A 96, 033621 (2017).

[58] Z. Birnbaum and B. A. Malomed, Families of spatial solitons in a two-channel waveguide with the cubic-quintic nonlinearity, Physica D 237, 3252 (2008).

[59] L. M. Chiofalo, S. Succi, and P. M. Tosi, Ground state of trapped interacting Bose-Einstein condensates by an explicit imaginary time algorithm, Phys. Rev. E 62, 7438 (2000).

[60] J. Yang and T. I. Lakoba, Accelerated imaginary-time evolution methods for the computation of solitary waves, Stud. Appl. Math. 120, 265 (2008).

[61] G. D. Peng, B. A. Malomed, and P. L. Chu, Soliton collisions in a model of a dual-core nonlinear optical fiber, Phys. Scripta 58, 149 (1998).

[62] N. Dror and B. A. Malomed, Symmetric and asymmetric solitons and vortices in linearly coupled two-dimensional waveguides with the cubic-quintic nonlinearity, Physica D 240, 526 (2011). 